\title{
Valuated Binary Tree: A New Approach in Study of Integers
}

\author{
WANG Xingbo \\ Department of Mechatronics \\ Foshan University, Foshan City, \\ Guangdong Province, PRC \\ wxbmail@msn.com
}

\begin{abstract}
The article first puts forward the concept of valuated binary tree; then makes a study on factors and multiples among the nodes of a binary tree that is valuated with odd numbers and obtains several new results that are valuable in study of integer division. The article also demonstrates that the approach of valuated binary tree is indeed practical and effectual because all its proofs and deductions are very elementary and intuitive, which is easier to understand and utilize.
\end{abstract}

Keywords: Binary tree, Valuated, Integer division, multiple

\section{INTRODUCTION}

Binary tree has been a familiar term in schoolbooks. Study of this non-linear data structure was a long time ago and no one in present day thinks it is worth to have a study on the binary tree. Compared to the fashionable bioinformatics or quantum computation, the binary tree is indeed an old thing. However if it is combined with an ever-older thing, it reveals younger and stronger traits.

With valuated binary tree, I obtain many new results when I study the old problems in elementary number theory. Hence I introduce the valuated binary tree in this article and use it to prove some theorems that disclose new properties of integer division. Readers can see that the proofs and deductions are very elementary and intuitive and it is easier to understand.

\section{Preliminaries}

This section gives definitions, notations and lemmas that are needed in later section. Also the studied question is presented in this section.

\subsection{Definitions, Notations and Question}

Definition 1. The floor function of a real number $x$ is denoted by $\lfloor x\rfloor$, and it fits $x-1<\lfloor x\rfloor \leq x$ or equivalently $\lfloor x\rfloor \leq x<\lfloor x\rfloor+1$.

The bibliography [1] lists properties of the floor function. Hence here I omit the details.

Definition 2. A valuated binary tree is such a binary tree that each of its node is assign a value. If all the nodes are assigned an integer number, then it is called an integer-valuated binary tree. We use symbol $\boldsymbol{T}$ to denote a binary tree.

Definition 3. Assign the root of a full binary tree with an odd number $N$, and assign the left and the right sons of the root with $2 N-1$ and $2 N+1$ respectively, and assign all the other nodes of the tree recursively by the previous regulation. This will obtain an $N$-rooted tree. For example, figure 1 demonstrates a 7-rooted tree. 


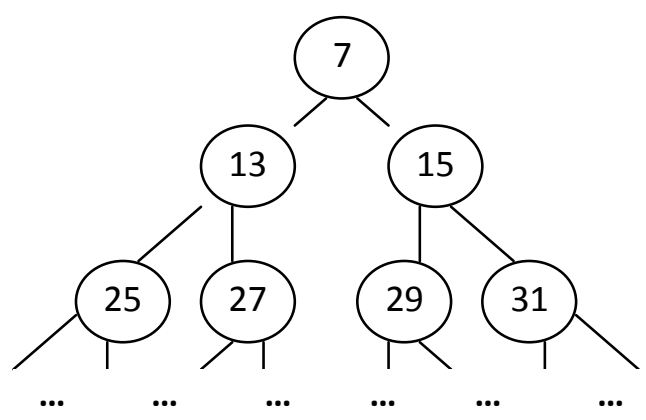

Fig.1. A 7-rooted binary tree

Question. Let us construct an $N$-rooted binary tree, where $N$ is an odd number bigger than one. For convenience, we denoted the root $N$ by $N_{0,0}$ and the node on the $j^{\text {th }}$ position of the $k^{\text {th }}$ level by $N_{k, j}$, where $k \geq 0$ and $0 \leq j \leq 2^{k}-1$, as shown in figure 2. Then under what condition $N_{0,0}$ can divide the other nodes?

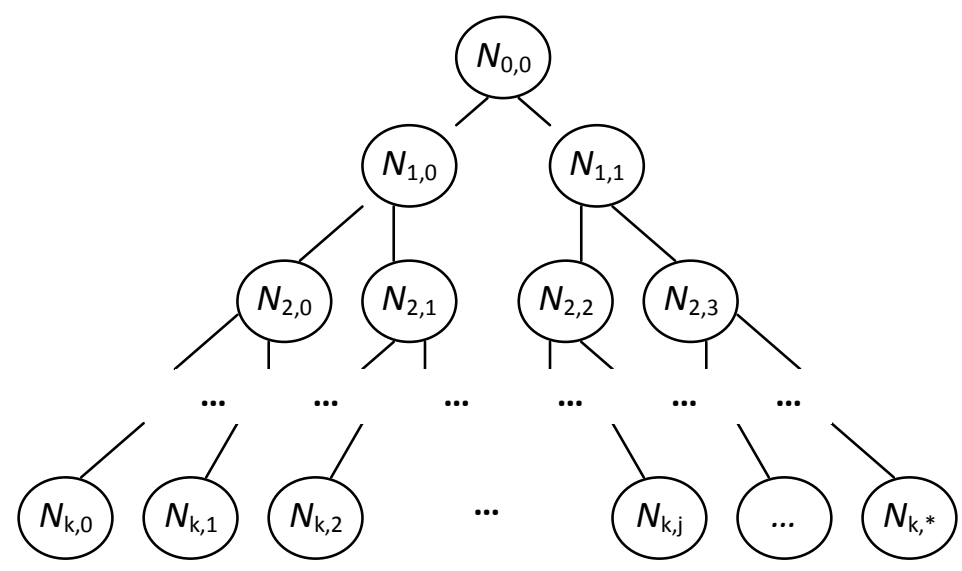

Fig.2. An $N_{0,0}$-rooted binary tree

\subsection{Lemmas}

Lemma 1. Let $p$ be a positive odd integer; then among $p$ consecutive positive odd integers there exists one and only one that can be divisible by $p$.

Proof. See in [2].

Lemma 2. Let $N>2$ be an integer; then $\left\lfloor\log _{2} N\right\rfloor \leq \log _{2}(1+N) \leq 1+\left\lfloor\log _{2} N\right\rfloor$.

Proof. See in [3].

Lemma 3. Let $q$ be a positive odd number, $S=\left\{a_{i} \mid i \in Z^{+}\right\}$be a set that is composed of consecutive odd numbers; if $a_{\alpha} \in S$ is a multiple of $q$, then so it is with $a_{\alpha+q}$.

Proof. Let $a_{\alpha}=\kappa q(\kappa>1)$; then it yields

$$
\left\{\begin{array}{l}
a_{\alpha+1}=\kappa q+2 \\
a_{\alpha+2}=\kappa q+4 \\
\cdots \ldots \\
a_{\alpha+q}=\kappa q+2 q=(\kappa+2) q
\end{array}\right.
$$

Lemma 4. Let $q$ be a positive odd number and $\mathrm{S}$ be a finite set that is composed of consecutive odd numbers; then $S$ needs at least $(n-1) q+1$ elements to have $n$ multiples of $q$.

Proof. Let $S=\left\{a_{0}, a_{1}, \ldots, a_{m-1}\right\}$ be the finite set that contains $n$ multiples of $q$. Obviously, $a_{0}$ is the smallest one and $a_{m-1}$ is the biggest one in the set. Now consider the first multiple of $q$. If $a_{0}$ is the 
first one, then $S$ needs at least $(n-1) q+1$ elements to have $n$ multiples of $q$, as shown in figure 3 . Otherwise it must hold $m>(n-1) q+1$.

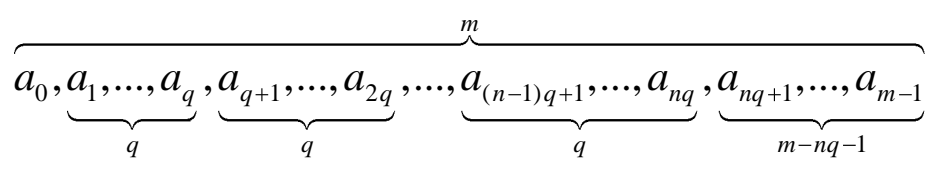

Fig.3. $\mathrm{n}$ multiples with $a_{0}$ being the first one

\section{Main Results AND Proofs}

Theorem 1. Let $\boldsymbol{T}$ be $N_{0,0}$-rooted binary tree. Then the following statements hold

(1) There is not a multiple of $N_{0,0}$ before the level $1+\left\lfloor\log _{2} N_{0,0}\right\rfloor$.

(2) There are exactly 2 multiples of $N_{0,0}$ on the level $1+\left\lfloor\log _{2} N_{0,0}\right\rfloor$; there are at least 2 multiples of $N_{0,0}$ on each level after the level $1+\left\lfloor\log _{2} N_{0,0}\right\rfloor$.

(3) There are at least 2 nodes that are not multiple of $N_{0,0}$ on each level from and after the level $1+\left\lfloor\log _{2} N_{0,0}\right\rfloor$.

(4) If $N_{0,0}$ and $N_{i, \omega}$ have a common factor d, then $N_{0,0}$ and $N_{i, 2^{i}-1-\omega}$ also have d to be their common factor, where $i>0,0 \leq \omega \leq 2^{i}-1$.

(5) On the same level, there is not a node that is a multiple of another one.

Proof. By definition 3, it is easy to obtain T's following properties.

(1) There are $2^{k}$ nodes on the $k^{\text {th }}$ level.

(2) Node $N_{k, j}$ is computed by

$$
\begin{aligned}
& N_{k, j}=2^{k} N_{0,0}-2^{k}+2 j+1 \\
& k=1,2, \ldots ; j=0,1, \ldots, 2^{k}-1
\end{aligned}
$$

(3) The two nodes, $N_{i, \omega}$ and $N_{i, 2^{i}-1-\omega}$, are at the symmetric position of the $i^{\text {th }}$ level and it holds

$$
N_{i, \omega}+N_{i, 2^{i}-1-\omega}=2^{i+1} N_{0,0}
$$

To analyses relationship between $N_{0,0}$ and $N_{k, j}$, we rewrite (2) by

$$
N_{k, j}=2^{k} N_{0,0}+\Delta(k, j), k=1,2, \ldots ; j=0,1,2, \ldots, 2^{k}-1
$$

Obviously, for a specified level, say the $k^{\text {th }}$ level, $\Delta(k, j)$ is a function of $j$. Table 1 lists change of $\mathrm{j}$ and the values of $\Delta(k, j)$. It can see that, when $j=0,1, \ldots, 2^{k-1}-1(k>0), \Delta(k, j)$ traverses the whole set $\left\{-1,-3, \ldots,-\left(2^{k}-1\right)\right\} \quad, \quad$ totally $2^{k-1}$ numbers; and when $j=2^{k-1}, 2^{k-1}+1,2^{k-1}+2, \ldots$, $2^{k}-2,2^{k}-1(k>0), \Delta(k, j)$ traverses $\left\{+1,+3, \ldots,+\left(2^{k}-1\right)\right\}$, totally $2^{k-1}$ numbers.

$$
\left\{\begin{array}{l}
\Delta(k, j) \in\left\{-1,-3, \ldots,-\left(2^{k}-1\right)\right\}, k>0, j \in\left\{0,1, \ldots, 2^{k-1}-1\right\} \\
\Delta(k, j) \in\left\{+1,+3, \ldots,+\left(2^{k}-1\right)\right\}, k>0, j \in\left\{2^{k-1}, 2^{k-1}+1, \ldots, 2^{k}-1\right\}
\end{array}\right.
$$


Table 1. Change of $j$ and values of $\Delta(k, j)$

\begin{tabular}{|c|c|c|}
\hline$j$ & $N_{k, j}$ & $\Delta(k, j)$ \\
\hline$j=0$ & $2^{k} N_{0,0}-2^{k}+1+0=2^{k} N_{0,0}-\left(2^{k}-1\right)$ & $-\left(2^{k}-1\right)$ \\
\hline$j=1$ & $2^{k} N_{0,0}-2^{k}+1+2=2^{k} N_{0,0}-\left(2^{k}-3\right)$ & $-\left(2^{k}-3\right)$ \\
\hline$j=2$ & $2^{k} N_{0,0}-2^{k}+1+4=2^{k} N_{0,0}-\left(2^{k}-5\right)$ & $-\left(2^{k}-5\right)$ \\
\hline$\ldots \ldots$ & $\ldots \ldots$ & $\ldots \ldots$ \\
\hline$j=2^{k-1}-2$ & $2^{k} N_{0,0}-2^{k}+1+2 \times\left(2^{k-1}-2\right)=2^{k} N_{0,0}-3$ & -3 \\
\hline$j=2^{k-1}-1$ & $2^{k} N_{0,0}-2^{k}+1+2 \times\left(2^{k-1}-1\right)=2^{k} N_{0,0}-1$ & +1 \\
\hline$j=2^{k-1}$ & $2^{k} N_{0,0}-2^{k}+1+2 \times 2^{k-1}=2^{k} N_{0,0}+1$ & +3 \\
\hline$j=2^{k-1}+1$ & $2^{k} N_{0,0}-2^{k}+1+2 \times\left(2^{k-1}+1\right)=2^{k} N_{0,0}+3$ & +5 \\
\hline$j=2^{k-1}+2$ & $2^{k} N_{0,0}-2^{k}+1+2 \times\left(2^{k-1}+2\right)=2^{k} N_{0,0}+5$ & $\ldots \ldots$ \\
\hline$\ldots \ldots$ & & $\ldots \ldots$ \\
\hline$j=2^{k-1}+2^{k-1}-2$ & $2^{k} N_{0,0}-2^{k}+1+2 \times\left(2^{k}-2\right)=2^{k} N_{0,0}+2^{k}-3$ & $+\left(2^{k}-3\right)$ \\
\hline$j=2^{k-1}+2^{k-1}-1$ & $2^{k} N_{0,0}-2^{k}+1+2 \times\left(2^{k}-1\right)=2^{k} N_{0,0}+2^{k}-1$ & $+\left(2^{k}-1\right)$ \\
\hline
\end{tabular}

Now it knows from (4) and (5) that $N_{0,0}$ can not divide $N_{k, j}$ when $N_{0,0}>2^{k}-1$ or $k<\log _{2}\left(1+N_{0,0}\right)$. By Lemma 2, we know $\log _{2}\left(1+N_{0,0}\right) \leq 1+\left\lfloor\log _{2} N_{0,0}\right\rfloor$; hence when $k<1+\left\lfloor\log _{2} N_{0,0}\right\rfloor$ or $k \leq\left\lfloor\log _{2} N_{0,0}\right\rfloor$, $N_{0,0}$ can not divide $N_{k, j}$. That is what statement (1) says.

Now consider the case $k \geq 1+\left\lfloor\log _{2} N_{0,0}\right\rfloor$. This time it holds $2^{k-1} \geq 2^{\left\lfloor\log _{2} N_{0,0}\right\rfloor}>2^{\log _{2} N_{0,0}-1}=\frac{1}{2} N_{0,0}$, namely $2^{k}>N_{0,0}$ or $2^{k}-1 \geq N_{0,0}$. Since $N_{0,0}>1$ is an odd number, it must be one of the element in the set $S=\left\{+3, \ldots,+\left(2^{k}-1\right)\right\}$. Namely, there exists a $j_{0} \in\left\{2^{k-1}, 2^{k-1}+1, \ldots, 2^{k}-1\right\}$ such that $N_{0,0}=\Delta\left(k, j_{0}\right)$ and it consequently holds $N_{0,0} \mid N_{k, j_{0}}$ and by (3) $N_{0,0} \mid N_{k, 2^{k}-1-j_{0}}$. Note that +1 is not an element of the set $S$, it shows that there must be a $j_{*} \in\left\{2^{k-1}, 2^{k-1}+1, \ldots, 2^{k}-1\right\}$ such that $N_{0,0} \neq \Delta\left(k, j_{*}\right)$, namely $N_{k, j_{*}}$ and $N_{k, 2^{k}-1-j_{*}}$ cannot be multiples of $N_{0,0}$. This validates statement (3).

Now we prove that on the level $k=1+\left\lfloor\log _{2} N_{0,0}\right\rfloor$ there exact 2 multiples of $N_{0,0}$. In fact, from $k=1+\left\lfloor\log _{2} N_{0,0}\right\rfloor$ we know $2^{k}=2^{\left.1+\log _{2} N_{0,0}\right\rfloor} \leq 2^{1+\log _{2} N_{0,0}}=2 N_{0,0}$, which says that the nodes on the level $k=1+\left\lfloor\log _{2} N_{0,0}\right\rfloor$ do not exceed $2 N_{0,0}$. Therefore there cannot be more than 2 multiples on the level according to Lemma 4 . Hence the statement (2) is sure.

Statement (4) naturally can be directly proved true by (4).

Take an arbitrary level, say the $k^{\text {th }}$ level. Note that the smallest and the biggest node on the level are $2^{k}\left(N_{0,0}-1\right)+1$ and $2^{k}\left(N_{0,0}+1\right)-1$ respectively, and $\frac{2^{k}\left(N_{0,0}+1\right)-1}{2^{k}\left(N_{0,0}-1\right)+1}<\frac{\left(N_{0,0}+1\right)}{\left(N_{0,0}-1\right)}<2$, it immediately knows that no node can be a multiple of the other one on the level. This is what the statement (5) says. And by now the whole theorem 1 is proved to be true.

\section{CONClusion}

It is of course a new approach to study integers with a binary tree. The research in this article shows that the new approach is both elementary and intuitive, and is better in digging out new properties of integers. Actually, I have dug out many other properties of integers and I will publish them in later articles. This article is the first one of the new approach, and the purpose of this article is to introduce the idea and the thought of the approach. I hope it can be concerned and better achievements can be obtained. 


\section{ACKNOWLEDGEMENTS}

The research work is supported by the national Ministry of science and technology under project 2013GA780052, Department of Guangdong Science and Technology under projects 2015A030401105 and 2015A010104011,, Foshan Bureau of Science and Technology under projects 2013AG10007, Special Innovative Projects 2014KTSCX156, 2014SFKC30 and 2014QTLXXM42 from Guangdong Education Department. The authors sincerely present thanks to them all.

\section{REFERENCES}

[1] Graham R L, Knuth D E and Patashnik O. "Integer Functions." Ch. 3 in Concrete Mathematics: A Foundation for Computer Science(2nd ed) , Addison-Wesley Professional;pp 67-101.

[2] WANG Xingbo. New Constructive Approach to Solve Problems of Integers' Divisibility, Asian Journal of Fuzzy and Applied Mathematics ,2014, 2(3):74-82

[3] WANG Xingbo.Some Supplemental Properties with appendix Applications of Floor Function,Journal of Science of Teachers' College and University(in Chinese),2014,34(3):7-9

\section{AUTHOR's BIOGRAPHY}

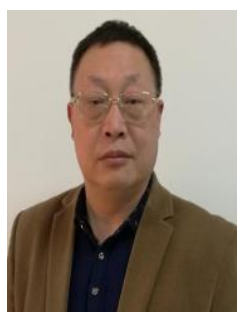

WANG Xingbo, was born in Hubei, China. He got his Master and Doctor's degree at National University of Defense Technology of China and had been a staff in charge of researching and developing $\mathrm{CAD} / \mathrm{CAM} / \mathrm{NC}$ technologies in the university. Since 2010, he has been a professor in Foshan University, still in charge of researching and developing $\mathrm{CAD} / \mathrm{CAM} / \mathrm{NC}$ technologies. Wang has published 8 books, over 80 papers and obtained more than 20 patents in mechanical engineering. 